\title{
The use of Chinese herbal products for nasopharyngeal carcinoma in Taiwan: A population-based study
}

Shih-Ting Tseng ${ }^{1, \#}$, Chun-Ting Liu ${ }^{1, \#}$, Bei-Yu Wu ${ }^{1}$, Yu-Chiang Hung ${ }^{1,2}$, Chung Y. $\mathrm{Hsu}^{3}$, Hsuan-Ju Chen ${ }^{4,5}$, Wen-Long $\mathrm{Hu}^{1,6,7, *}$

${ }^{1}$ Department of Chinese Medicine, Kaohsiung Chang Gung Memorial Hospital and School of Traditional Chinese Medicine, Chang Gung University College of Medicine, Kaohsiung, Taiwan

No. 123, Dapi Rd., Niaosong Dist., Kaohsiung 833, Taiwan

${ }^{2}$ School of Chinese Medicine for Post Baccalaureate, I-Shou University, Kaohsiung,

Taiwan

No. 1, Sec. 1, Syuecheng Rd., Dashu District, Kaohsiung 84001, Taiwan

${ }^{3}$ Graduate Institute of Biomedical Sciences, China Medical University, Taichung,

Taiwan

No. 91, Hsueh-Shih Rd., Taichung 40402, Taiwan

${ }^{4}$ Management Office for Health Data, China Medical University Hospital, Taichung, Taiwan

No. 2, Yuh-Der Rd., Taichung 40447, Taiwan 
${ }^{5}$ College of Medicine, China Medical University, Taichung, Taiwan

No. 91, Hsueh-Shih Rd., Taichung 40402, Taiwan

${ }^{6}$ Kaohsiung Medical University College of Medicine, Kaohsiung, Taiwan

No. 100, Shihcyuan 1st Rd., Sanmin Dist., Kaohsiung 807, Taiwan

${ }^{7}$ Fooyin University College of Nursing, Kaohsiung, Taiwan

No. 151, Chinhsueh Rd., Ta-liao Dist., Kaohsiung 831, Taiwan

Running title: Chinese Herbal Products for NPC

This article contains 27 pages, 4 figures, and 5 tables.

${ }^{\#}$ These authors contributed equally to this work

* Correspondence to: Wen-Long $\mathrm{Hu}$

No. 123, Dapi Rd., Niaosong Dist., Kaohsiung 83301, Taiwan.

E-mail: oolonghu@gmail.com; oolonghu@cgmh.org.tw; Tel.: +886-731-7123, ext.

2334; Fax: +886-731-7123, ext. 2335 
Abstract: In most countries, the incidence of nasopharyngeal carcinoma (NPC) is no more than 1 per 100,000 for both men and women; however, it is much higher for men and women in Taiwan. The use of traditional Chinese medicine (TCM) as complementary and alternative medicine for the treatment of NPC and its treatment-related side effects has been increasing. The National Health Insurance (NHI) covers $99.6 \%$ of Taiwan's residents. In the present population-based cohort study, we aimed to investigate the pattern of utilization of Chinese herbal products (CHPs) for NPC from 2001 through 2011 in Taiwan. We identified a total of 30294 patients with newly diagnosed NPC from the Registry for Catastrophic Illnesses Patient Database (RCIPD). Descriptive statistics and multiple logistic regression analysis were employed to estimate the adjusted odds ratios (aORs) for CHP utilization. From 2001 through 2011, 17816 patients aged $\geq 20$ years were newly diagnosed with NPC. Of these, 4749 patients used TCM outpatient services for NPC treatment. TCM users were more likely to be women, young, residents of Central Taiwan, and white-collar workers. The most commonly prescribed formula CHP was Gan-Lu-Yin, followed by Xin-Yi-Qing Fei-Tang and Shan-Shen-Mai-Men-Dong-Tang. The most commonly prescribed single CHP was Hedyotis diffusa, followed by Radix Scrophulariae and Radix Ophiopogonis. These findings provide information regarding personalized therapies for NPC and can 
promote further clinical experiments and pharmacological research on CHPs for NPC treatment in Taiwan. Further well-designed randomized controlled studies and basic mechanistic studies should assess the safety and effectiveness of CHPs for NPC treatment.

Keywords: nasopharyngeal carcinoma; traditional Chinese medicine; Chinese herbal products; complementary and alternative medicine; Gan-Lu-Yin 


\section{Introduction}

Nasopharyngeal carcinoma (NPC) is a malignancy arising from the nasopharynx, most commonly the posterolateral nasopharynx, pharyngeal recess, or fossa of Rosenmüller. The most common presentation is a palpable neck lump, with approximately $60 \%$ patients seeking medical attention when this occurs. Such lumps develop because of metastatic disease in the cervical lymph nodes. In endemic regions such as China, Hong Kong, and Singapore, a Chinese adult man seen with large, bulky bilateral nodes is generally considered to have NPC or lymphoma. Although $60 \%$ patients with NPC initially present with a neck lump caused by nodal metastasis, at least $80 \%$ patients are diagnosed with positive nodes (N1, N2, or N3) [1]. Men are two to three times more likely to develop NPC than are women, and the peak age of occurrence is between 50 and 60 years [2]. 
(a)

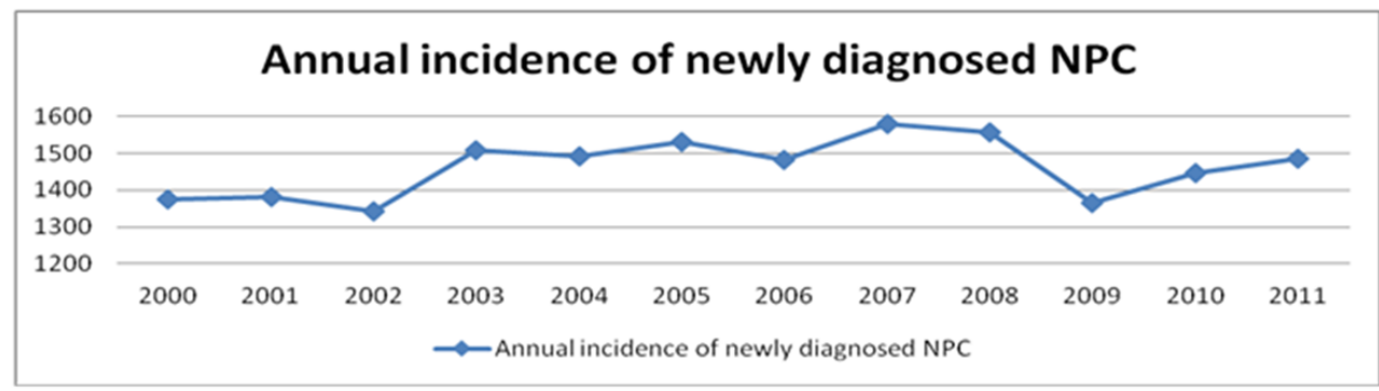

(b)

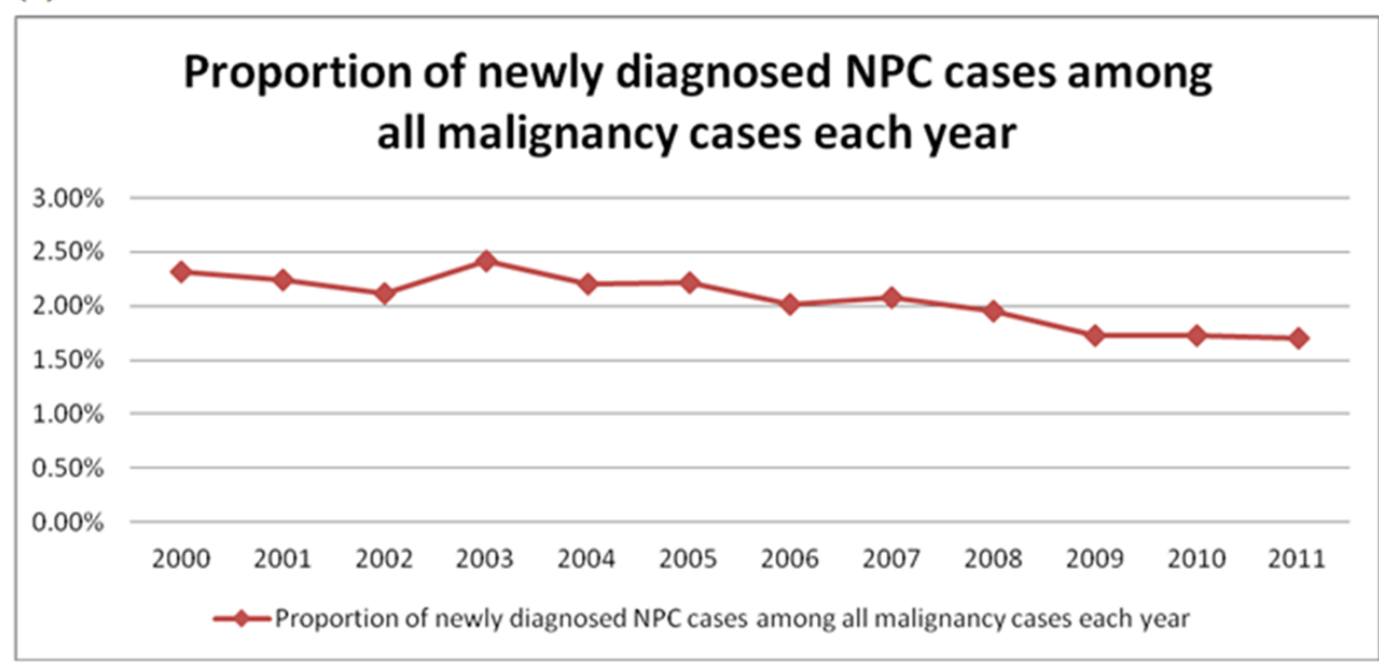

Fig. 1a Annual incidence of newly diagnosed nasopharyngeal carcinoma (NPC) from 2000 through 2011 in Taiwan.

The graph shows that approximately 1300-1600 individuals were diagnosed with NPC each year.

Fig. 1b Proportion of newly diagnosed NPC cases among all cases of malignancy each year from 2000 through 2011 in Taiwan

The graph shows that newly diagnosed cases of NPC accounted for approximately $2 \%$ of all malignancy cases each year.

The incidence of NPC is reportedly less than 1 per 100,000 for both men and women in most countries; however, it is $20-30$ per 100,000 men and $15-20$ per 100,000 women in Hong Kong [3]. NPC is an infection-related cancer that is strongly driven by the Epstein-Barr virus. The occurrence of this infection is strongly associated with genetics, particularly in South China, Hong Kong, and Taiwan [4, 5]. 
From 2001 through 2011, approximately 1300-1600 individuals were newly diagnosed with NPC in Taiwan (Figure 1a), with NPC accounting for approximately $2 \%$ of all cases of malignancies (Figure 1b) during this period. In fact, NPC is the tenth most common cancer among men in Taiwan [6,7].

Interest in complementary and alternative medicine (CAM) is increasing among not only patients looking for help but also healthcare providers studying the effectiveness of various therapies and management techniques. Up to $52 \%$ cancer patients use one or more CAM treatments, and over $80 \%$ patients use CAM concurrently with chemotherapeutic agents during the initial phases of cancer treatment [8-10]. Traditional Chinese medicine (TCM) is the most common CAM in our clinical practice. Considering that different stages of NPC are characterized by various clinical features and complications before and after conventional treatments, TCM doctors typically provide treatment using different combinations of Chinese herbs. In Taiwan, Chinese herbal products (CHPs) have been listed under the National Health Insurance (NHI) program since 1995. NHI covers $99.6 \%$ of Taiwan's residents. However, there is a lack of large-scale statistical data on the clinical usage of TCM in NPC. Therefore, we aimed to investigate the utilization of CHPs for NPC from 2001 through 2011 in Taiwan by exploring the demographic characteristics of patients and the patterns of CHP prescriptions. While demographic data can provide relevant 
information for clinical pharmaceutical and epidemiological studies, data regarding prescription patterns can provide information regarding the monitoring, evaluation, and modification of medical services and facilitate the establishment of reference data concerning individualized therapies for NPC.

\section{Methods}

\section{Data sources}

The National Health Insurance (NHI) program of Taiwan is a universal insurance program established in 1995, reforming from 13 insurance-related systems and providing coverage for $99.9 \%$ Taiwanese citizens by the end of 2014 . The NHI Research Database (NHIRD) has been managed, maintained, and released by the National Health Research Institute (NHRI) in Taiwan. In this population-based cohort study, we obtained data from the Registry for Catastrophic Illnesses Patient Database (RCIPD), which is a part of NHIRD. RCIPD contains claims data from insurers diagnosed with one of 30 categories of major diseases (cancer, chronic mental illness, end-stage renal disease, several autoimmune diseases, etc.) who require long-term care and are exempted from co-payment. Registered data include demographic data, the date of visit or hospitalization, drug prescriptions, and diagnoses coded in the format of the International Classification of Disease, Ninth Revision, Clinical 
Modification (ICD-9-CM). For the protection of insurer privacy, all potentially identifying information was encrypted. The study was approved by the Ethics Review Board of China Medical University (CMUH1O4-REC2-115).

\section{Study population}

We identified 30294 patients with newly diagnosed NPC (ICD-9-CM 147) from RCIPD. Patients with missing information on age or sex $(n=14)$, those diagnosed with NPC before the end of $1999(n=12302)$, and those aged <20 years $(n=162)$ were excluded. Eventually, 17816 patients were included in the study and further divided into TCM users $(n=9577)$ and TCM nonusers $(n=8239$; Figure 2$)$. The former were defined as individuals who used TCM outpatient services at least once during the study period, while the latter were defined as individuals who never visited TCM clinics after the first NPC diagnosis.

Evaluated demographic factors included sex, age (20-29, 30-39, 40-49, 50-59, and $\geq 60$ years), occupational status (white collar, blue collar, and others), and residential areas area (northern Taiwan, central Taiwan, southern Taiwan, and eastern Taiwan and offshore islands).

\section{Statistical analysis}


Descriptive statistics for the TCM users and TCM nonusers are presented as

means and standard deviations (SD) for continuous variables and numbers and percentages for categorical variables. Differences in the distribution of these statistics between the two groups were assessed using Student's t-test for continuous variables and Pearson's chi-square test for categorical variables. Multiple logistic regression analysis was performed to determine the correlation between demographic data (sex, age, occupational status, and residential area) and TCM use. The logistic regression model produced odds ratios (ORs) and corresponding 95\% confidence intervals (CIs). Adjusted ORs (aORs) were used to predict patients with higher odds of using TCM. All statistical analyses were performed using SAS software, version 9.4 (SAS Institute, Cary, NC, USA). All P-values were derived from two-sided tests, with the significance level set at 0.05 .

\section{Results}

The patient recruitment flowchart is presented in Figure 2. In total, 17816 patients aged $\geq 20$ years were newly diagnosed with NPC from 2001 through 2011 . From these, 4749 patients used TCM outpatient services for NPC treatment. The majority of TCM users were men (71.3\%), and TCM users were younger than TCM nonusers (40.9 versus 52.9 years). Moreover, a larger proportion of TCM users tended 
to be white-collar workers and residents of northern Taiwan. Table 1 shows aORs and 95\% CIs derived from multiple logistic regression analysis. Women (aOR, 1.47; 95\% CI, 1.37-1.58); patients aged 20-29 (aOR, 2.33; 95\% CI, 1.96-2.77), 30-39 (aOR, 2.15; 95\% CI, 1.95-2.37), 40-49 (aOR, 1.80; 95\% CI, 1.65-1.95), or 50-59 (aOR, $1.52 ; 95 \% \mathrm{CI}, 1.39-1.65)$ years; and residents of central (aOR, 1.49; 95\% CI, 1.37-1.62) and southern (aOR, 1.12; 95\% CI, 1.05-1.21 Taiwan) were more likely to utilize TCM services, whereas blue-collar workers (aOR, 0.88; 95\% CI, 0.83-0.94) and residents of eastern Taiwan and offshore islands (aOR, 0.80; 95\% CI, 0.70-0.91) were less likely to use TCM services (Table 1). 


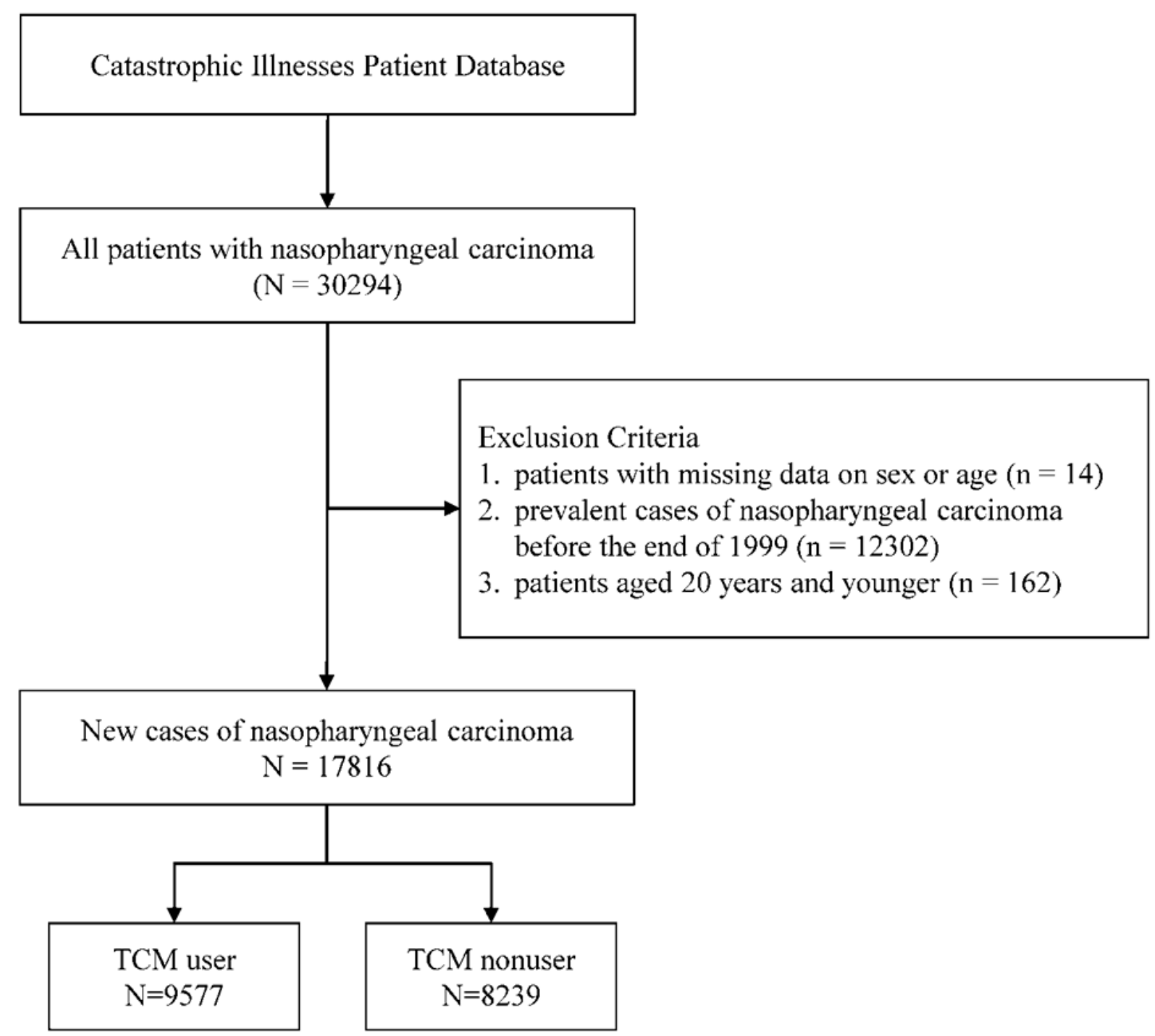

Figure 2. Flowchart of recruitment of subjects from Catastrophic Illnesses Patient Database during 2000-2011 in Taiwan.

Abbreviation: TCM, traditional Chinese medicine. 
Table 1. Demographic characteristics and results of multiple logistic regression analysis for patients newly diagnosed with nasopharyngeal carcinoma from 2001 through 2011 in Taiwan

$$
\begin{array}{lll}
\text { TCM nonusers } & \text { TCM users } & \text { p-value }
\end{array}
$$

\begin{tabular}{|c|c|c|c|c|c|c|c|}
\hline Characteristics & $\mathrm{N}$ & $\%$ & $\mathrm{~N}$ & $\%$ & & Crude & Adjusted $^{\dagger}$ \\
\hline No. of patients & 8239 & & 9577 & & & & \\
\hline TCM for nasopharyngeal & & & 4749 & & & & \\
\hline carcinoma & & & & & & & \\
\hline Sex & & & & & $<0.001$ & & \\
\hline Women & 1785 & 21.7 & 2749 & 28.7 & & $1.46(1.36-1.56)^{* * *}$ & $1.47(1.37-1.58)^{* * *}$ \\
\hline Men & 6454 & 78.3 & 6828 & 71.3 & & 1.00 & 1.00 \\
\hline
\end{tabular}

nasopharyngeal carcinoma,

years

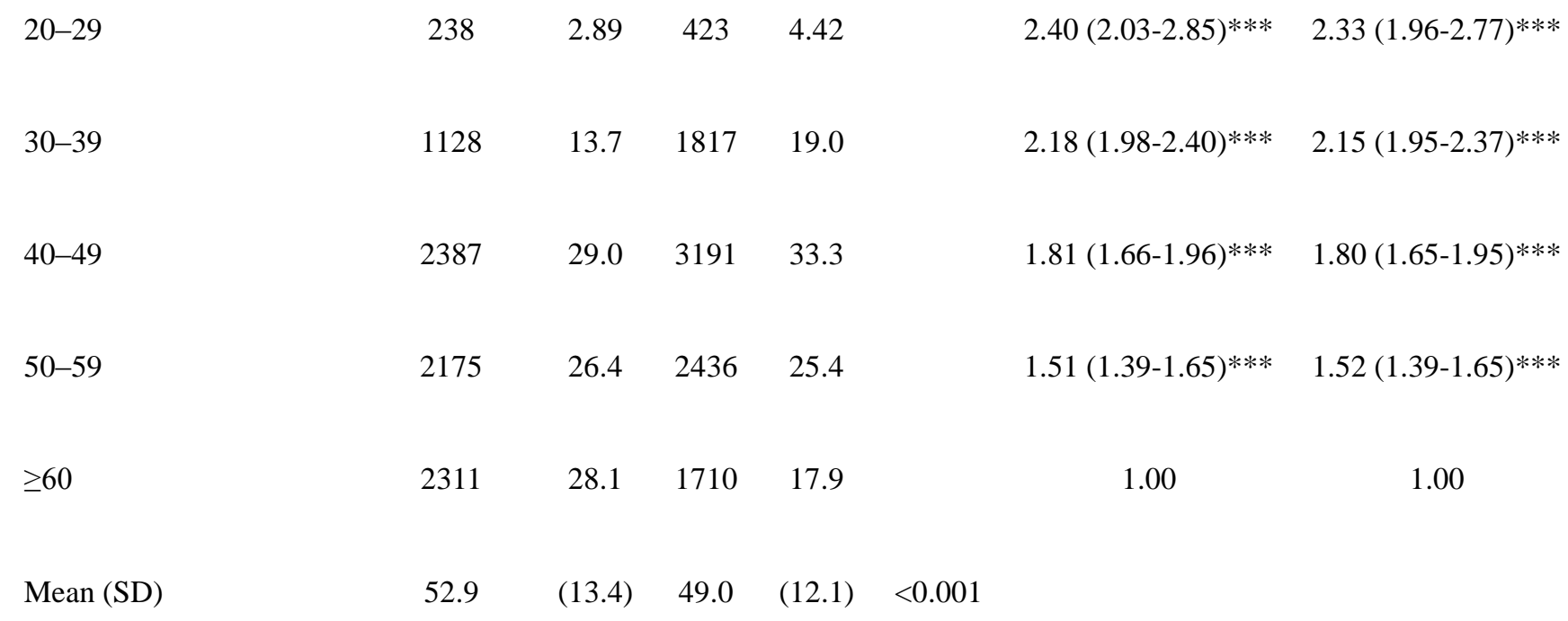


Occupational status

White collar

Blue collar

Others

Residential areas

Northern area

Central area

Southern area

Eastern area and offshore
3943

47.

3171

1125

1049

11.0

$0.72(0.65-0.80) * * *$

$0.74(0.67-0.81) * * *$

$<0.001$

3667

1363

$16.5 \quad 2105$

22.0

2628

31

3022

31.6

581

7.05

461

4.81

islands

TCM, traditional Chinese medicine; SD, standard deviation; OR, odds ratio; CI, confidence interval

${ }^{\dagger}$ Model adjusted for sex, age (categorical), occupational status, and area

$* \mathrm{p}<0.05, * * \mathrm{p}<0.01, * * * \mathrm{p}<0.001$

The average number of CHPs in a single prescription for TCM users was 6.56

(Figure 3), with the majority of prescriptions containing six herbs, followed by seven and five. 


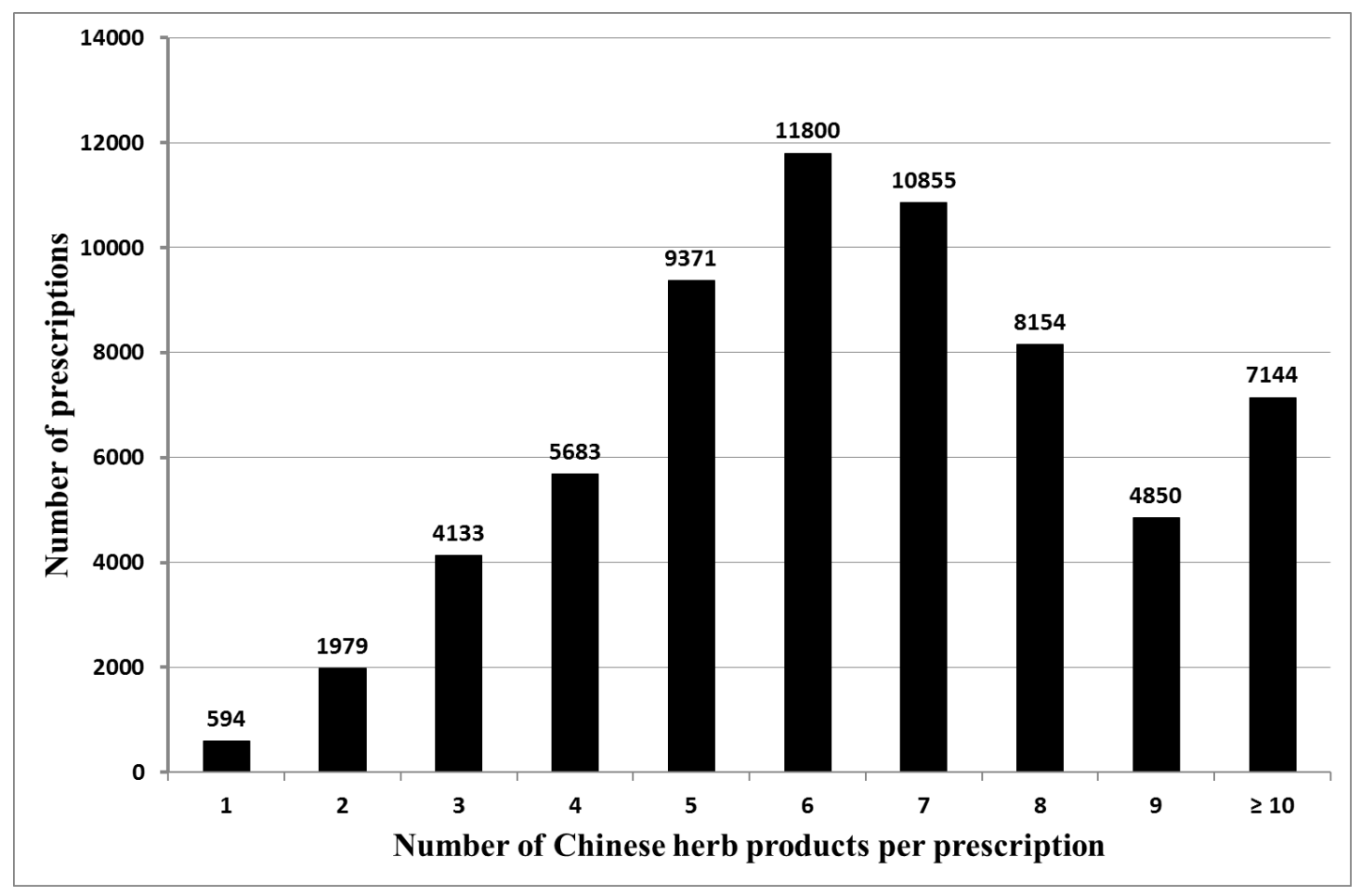

Figure 3. Distribution of the number CHP in one prescription. The most common prescriptions of CHP combinations or single Chinese herbs contained 5 to 7 herbs. There was an average of 6.56 Chinese herbs in a single prescription for patients with nasopharyngeal carcinoma.

Abbreviation: CHP, Chinese herb products.

Tables 2 and 3 show details, including mechanisms of action or effects, of the most commonly prescribed formula and single CHPs. Gan-Lu-Yin was the most frequently prescribed formula CHP, followed by Xin-Yi-Qing-Fei-Tang,

Sha-Shen-Mai-Men-Dong-Tang, Zhi-Bai-Di-Huang-Wan, and

Shan-Zhong-Kui-Jain-Tang. The most commonly prescribed single herb was

Bai-Hua-She-She-Cao, followed by Xuan Shen, Mai-Men-Dong, Dan Shen, and Ban-Zhi-Lian (Tables 2 and 3). 
Table 2. Possible mechanisms of action or effects of frequently used formula or single CHPs for nasopharyngeal carcinoma in Taiwan (2001 through 2011)

Top three formula and

$$
\text { Components }
$$

single CHPs

\section{Gan-Lu-Yin}

Rehmannia glutinosa,

Rehmannia glutinosa

Preparata, Herba Dendrobii,

Asparagus cochinchinensis,

Radix Ophiopogonis,

Artemisia scoparia Waldst,

Radix scutellariae, Citrus

aurantium L., Eriobotrya

japonica, Radix Glycyrrhizae

Xin-Yi-Qing-Fei-Tang $\quad$ Magnolia liliflora Desr.,

Lilium brownie, Anemarrhena

asphodeloides, calcium

sulphate dehydrate,

Eriobotrya japonica, Rhizoma

Cimicifugae, Radix
Clears heat, nourishes Yin,

generates fluids

oral cancer cells through the $\mathrm{NF}-\kappa \mathrm{B}$,

AKT, and ERK-dependent pathways

Clears heat from the lungs and

Mechanism unknown pathways [11]

Suppresses TNF- $\alpha$ levels in human

Suppresses vascular smooth muscle

cell migration by inhibiting matrix

metalloproteinase-2/9 through the

PI3K/AKT and ERK signaling stomach, unblocks the orifices

(particularly nose) 
Ophiopogonis, Fructus

Gardeniae, Radix scutellariae,

Radix Glycyrrhizae

Shan-Shen-Mai-Men-

Dong-Tang

Bai Hua She She Cao
Radix Adenophorae, Radix

Ophiopogonis, Rhizoma

Polygonati Odorati, Folium

Mori, Radix Trichosanthis,

Semen Lablab Album, Radix

Glycyrrhizae

Hedyotis diffusa

fire toxins and abscesses

Scrophularia ningpoensis

and stomach, eliminates

dryness, generates fluids
Clears heat from the lungs and

stomach, nourishes the lungs

Clears heat, strongly relieves

Inhibitory effect on the c-Jun

N-terminal kinase (JNK) signaling

pathway [16]

Clears heat and cools the blood, Iridoid and phenylpropanoid

nourishes Yin, relieves toxicity glycosides from Scrophularia 
ningpoensis Hemsl and their

$\alpha$-glucosidase inhibitory activities [17]

Disrupts focal adhesion and causes

detachment-induced apoptosis in

malignant cancer cells [18]

Inhibits ventricular remodeling

induced by hypertension in rats [19]

Mai Men Dong

Radix Ophiopogonis

Moistens the lungs, nourishes

Induces in vitro and in vivo

Yin, nourishes the stomach Yin

RIPK1-dependent and

and generates fluids, clears the

caspase-independent apoptotic death

heart and eliminates irritability

in cancer cells [20]

Decreases IL-6 production [21]

The products are listed in order of their frequency of use.

CHPs, Chinese herbal products; PI3K/AKT, phosphoinositide-3-kinase/protein kinase B; ERK, extracellular signal-regulated kinase-dependent; TNF- $\alpha$, tumor necrosis factor alpha; NF- $\kappa \mathrm{B}$, nuclear factor-kappa beta; RIPK1, receptor-interacting serine/threonine-protein kinase 1 
Table 3. Top ten formula and single CHPs prescribed by TCM physicians for the treatment of nasopharyngeal carcinoma in Taiwan (2001 through 2011; total number of CHPs, $\mathrm{n}=423545$ )

\begin{tabular}{|c|c|c|c|}
\hline Formula CHPs & Number (\%) & Single CHPs & Number $(\%)$ \\
\hline Gan-Lu-Yin & $12651(2.99)$ & Hedyotis diffusa & $13666(3.23)$ \\
\hline Xin-Yi-Qing-Fei-Tang & $6428(1.52)$ & Radix Scrophulariae & $9469(2.24)$ \\
\hline Shan-Shen-Mai-Men-Dong-Tang & $3912(0.92)$ & Radix Ophiopogonis & $7773(1.84)$ \\
\hline Zhi-Bai-Di-Hung-Wan & $3858(0.91)$ & Salvia miltiorrhiza & $7031(1.66)$ \\
\hline Shan-Zhong-Kui-Jain-Tang & $2891(0.68)$ & Scutellariae Barbatae & $6062(1.43)$ \\
\hline Mai-Men-Dong-Tang & $2845(0.67)$ & Herba Dendrobii & $5831(1.38)$ \\
\hline Chang-Er-Zi-San & $2786(0.66)$ & Bulbus Fritillariae & $5120(1.21)$ \\
\hline Jia-We-Xiao-Yao-San & $2719(0.64)$ & Radix Trichosanthis & $4954(1.17)$ \\
\hline Yi-Guan-Jian & $2620(0.62)$ & Radix Puerariae & $4752(1.12)$ \\
\hline Bu-Zhong-Yi-Qi-Tang & $2539(0.60)$ & Radix Rehmanniae & $4621(1.069)$ \\
\hline
\end{tabular}

TCM, traditional Chinese medicine; CHPs, Chinese herbal products

The top five common combinations comprising two formula CHPs and those

comprising two single CHPs are shown in Table 4, while the top five combinations comprising three formula CHPs are shown in Table 5. 
Table 4. Top five combinations comprising two formula CHPs or two single

CHPs prescribed for nasopharyngeal carcinoma in Taiwan (2001 through 2011;

total number of CHPs, $\mathrm{n}=423545$ )

\begin{tabular}{cc}
\hline Two-formula-CHPs & Number (\%) \\
\hline Gan-Lun-Yin and Zhi-Bai-Di-Huang-Wan & $1147(0.27)$ \\
Gan-Lun-Yin and Xin-Yi-Qing-Fei-Tang & $894(0.21)$ \\
Jia-Wei-Xia-Yao-San and Gan-Lun-Yin & $617(0.15)$ \\
Gan-Lun-Yin and Ma-Zi-Ren-Wan & $564(0.13)$ \\
Gan-Lun-Yin and Cang-Er-Zi-San & $514(0.12)$ \\
Two-single-CHPs & Number (\%) \\
\hline Radix Rehmanniae and Radix Ophiopogonis & $5003(1.18)$ \\
\hline Salvia miltiorrhiza and Hedyotis diffusa & $2900(0.68)$ \\
\hline
\end{tabular}

CHPs, Chinese herbal products 
Table 5. Top five combinations comprising three formula CHPs prescribed for nasopharyngeal carcinoma in Taiwan (2001 through 2011; total number of CHPs, $\mathrm{n}=$ 423545)

Three-formula-CHPs Number $(\%)$

Ma-Zi-Ren-Wan, Zhu-Ling-Tang, and Long-Dan-Xie-Gan-Tang $144(0.03)$

Shu-Jing-Huo-Xue-Tang, Dang-Gui-Nian-Tong-Tang, and $103(0.02)$

Du-Huo-Ji-Sheng-Tang

Chai-Ge-Jie-Ji-Tang, Shu-Jing-Huo-Xue-Tang, and $73(0.02)$

Dang-Gui-Nian-Tong-Tang

Liu-Wei-Di-Huang-Wan, Tian-Wang-Bu-Xin-Dan, and $72(0.02)$

Suan-Zao-Ren-Tang

Chai-Ge-Jie-Ji-Tang, Shu-Jing-Huo-Xue-Tang, and $72(0.02)$

Du-Huo-Ji-Sheng-Tang

CHPs, Chinese herbal products

\section{Discussion}

In this nationwide cohort study of CHP use for NPC treatment from 2001 through 2011 in Taiwan, we observed that women were more likely to use TCM than were men. Moreover, TCM users were younger than nonusers, probably because younger 
patients readily accept a variety of treatments. Patients in central Taiwan showed a higher OR for TCM use than did those in southern and Taiwan and northern Taiwan, possibly because central Taiwan has the highest density of TCM clinics in Taiwan. Therefore, TCM services are easily available for residents of central Taiwan. The allocation of TCM services could have affected the TCM-seeking behavior of patients. Our study results also showed that white-collar workers were more likely to use TCM. White-collar workers are generally richer than blue-collar workers, so they are able to afford additional treatments. Our results were in accordance with a previous study of TCM use by adult patients with cancer [22]. The demographic characteristics of NPC patients reported in this study may help public health policymakers and clinicians in making informed decisions regarding the distribution of TCM supplements in the different regions in Taiwan.

Conventional treatments for NPC include surgery, radiation therapy (RT), and chemotherapy or target therapy. Currently, RT is the mainstay of treatment for NPC because the primary tumor is not easily amenable to surgical resection in most cases [23]. Our data from NHI (shown in Figure 4) also showed that RT was the most common treatment strategy used for patients with NPC from 2001 through 2011 in Taiwan. However, RT often causes several side effects such as mucositis, xerostomia, dysphagia, dermatitis, weight loss, and malnutrition. Among these, xerostomia is one 
of the most common acute and long-term side effects that is primarily induced by RT and is dependent on the cumulative radiation doses to the head and neck region [1]. In recent years, the use of TCM in combination with conventional cancer therapy has become more widespread. NPC patients receiving conventional anticancer therapies combined with TCM treatment reportedly exhibited increased survival rates, enhanced tumor responses, an improved Karnofsky performance status, lower side effect rates, and better immunostimulation [24] Because NPC and its treatment can cause many complications, the goal of TCM is to not only try and cure the cancer but also prevent unwanted complications during conventional treatments.

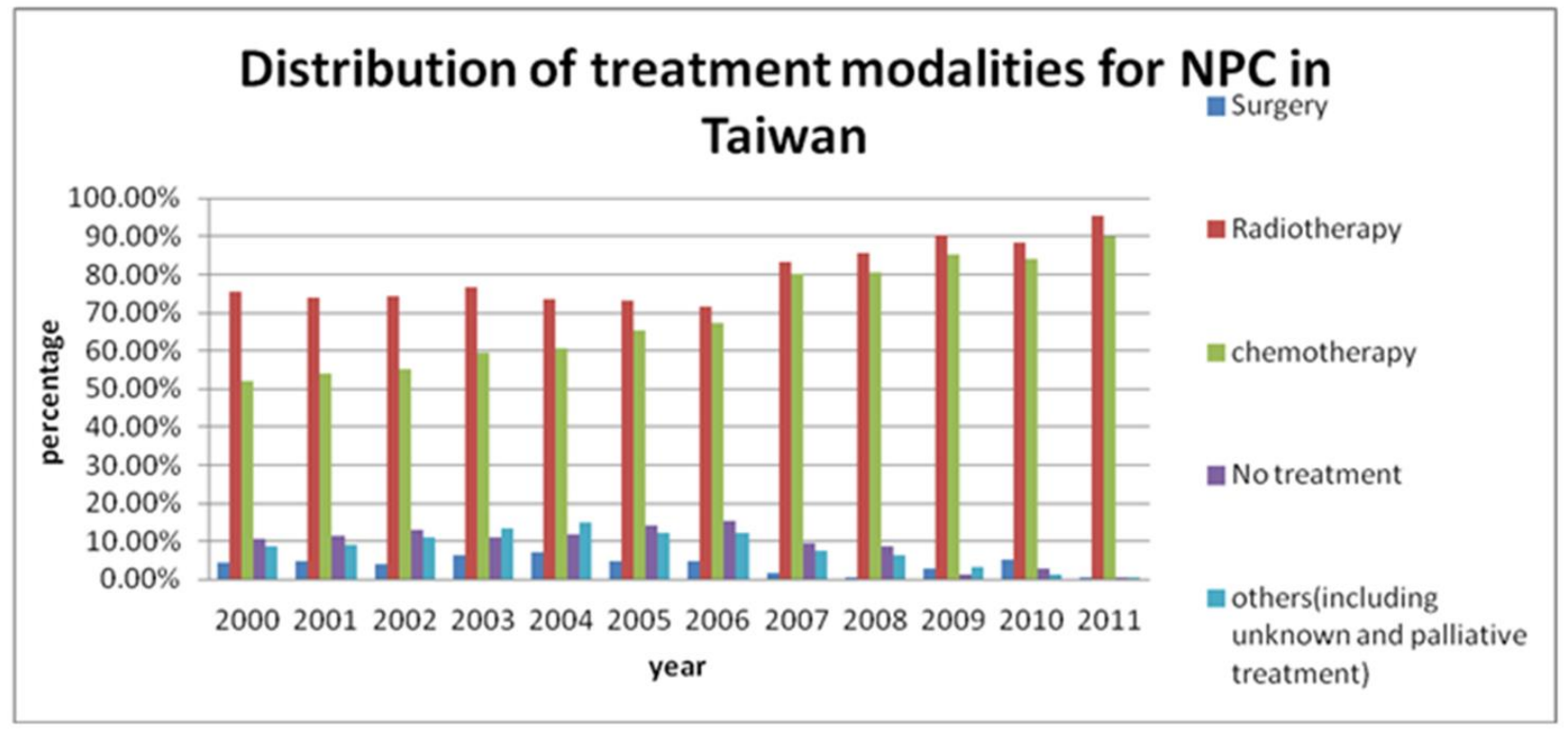

Figure 4. Distribution of treatment modalities for nasopharyngeal carcinoma (NPC) from 2000 through 2011 in Taiwan

The graph shows that radiotherapy was the most common treatment modality throughout the study period, with an increase in the number of patients over time. In addition, the number of patients with no treatment can be seen to decrease over time. 
We found that the most commonly prescribed formula CHP for NPC treatment was Gan-Lu-Yin, followed by Xin-Yi-Qing-Fei-Tang and Shan-Shen-Mai-Men-Dong-Tang. The prescription pattern indicated that TCM physicians usually formulated personalized therapies according to the presenting symptoms and signs. Gan-Lu-Yin is used to nourish yin and clear heat [25]. It is clinically used to treat gingivitis, conjunctivitis, mucositis, and stomatitis. A previous study showed that modified Gan-Lu-Yin, alone or in combination with other TCM formulae, could reduce the symptoms of dry mouth and treat acute mucositis caused by RT [26]. Several other studies reported that some single herbs within Gan-Lu-Yin had antitumor activities via the inhibition of cell proliferation and inflammation [27-29]. In one study, Gan-Lu-Yin was found to inhibit the migration of vascular smooth muscle cells (VSMCs) and block injury-induced neointimal hyperplasia without apoptosis by inhibiting matrix metalloproteinase (MMP)-2/9 through the phosphoinositide-3-kinase (PI3K)/protein kinase B (AKT) and extracellular signal-regulated kinase-dependent (ERK) signaling pathways [11]. This suggested that Gan-Lu-Yin could inhibit angiogenesis, which in turn inhibits tumor growth $[11,30]$. Gan-Lu-Yin was also reported to suppress tumor necrosis factor alpha (TNF- $\alpha$ ) in human oral cancer cells through nuclear factor-kappa B (NF-kB), AKT, and extracellular signal-regulated kinase-dependent (ERK) pathways [12]. In summary, 
Gan-Lu-Yin can be used as an adjuvant treatment to not only reduce RT-related side effects but also cure the cancer.

Xin-Yi-Qing-Fei-Tang, the second most commonly prescribed formula CHP in our study, clears heat from the lungs and stomach and unblocks the nasal orifices. In TCM practice, Xin-Yi-Qing-Fei-Tang is often appropriate for patients with yellow sputum; a stuffy nose; and thick, sticky, and yellow nasal discharge due to upper respiratory tract infection or allergies [31]. It is also the most common herbal formula used for children with allergic rhinitis and adults with chronic rhinosinusitis [31, 32]. Scutellaria baicalensis Georg (Huang Chin), an ingredient of Xin-Yi-Qing-Fei-Tang, was reported to have anticancer effects in several malignancies. It can induce cell cycle arrest as well as suppress cancer cell colony formation and migration [33-36]. NPC patients often exhibit yellow, thick, and sticky mucus due to upper respiratory tract infection or inflammation while receiving anticancer treatment. This could be the reason why Xin-Yi-Qing-Fei-Tang was such a commonly prescribed combination in our study.

Shan-Shen-Mai-Men-Dong-Tang is used to clear and nourish the lungs and stomach, engender fluid, repair damaged cells, and control the symptoms of dryness associated with cancer treatment [13]. One study by Sung and Dong (2001) in China also showed that Shan-Shen-Mai-Men-Dong-Tang could improve oral dryness and 
resolve difficulties in chewing and swallowing in patients with head and neck cancer who received chemoradiotherapy [37]. In addition, Shan-Shen-Mai-Men-Dong-Tang, the third most common formula CHP in our study, may strengthen the patient's immune system, because it was found to significantly increase the Zn levels in urine samples from patients with head and neck cancer [13]. It has been previously shown that $\mathrm{Zn}$ plays a significant role in the metabolic response to injury and wound healing [38], and increasing the Zn levels may enhance the healing process for patients with RT-induced mucositis and dermatitis.

With regard to single CHPs, the most common one in our study was Hedyotis diffusa, followed by Radix Scrophulariae and Radix Ophiopogonis.

Our findings were consistent with those of a previous NHI survey, where Hedyotis diffusa was the most commonly prescribed single herb for patients with cancer [39]. This CHP is used to clear heat, strongly relieve fire toxins, and abscesses. It exhibits several biological activities such as anticancer, antioxidative, anti-inflammatory, and neuroprotective effects $[15,40]$. Hedyotis diffusa also showed suppressive effects on the aggressive phenotypes of laryngeal squamous carcinoma cells, induced cell cycle arrest and apoptosis, and inhibited cancer cell invasion via inhibition of B-cell lymphoma 2 (Bcl-2), MMP-2, and $\mu \mathrm{PA}$, which are involved in cell growth, survival, and invasion $[14,15]$. In addition, it inhibited inducible nitric oxide synthase and 
interleukin-1 $\beta$ expression in a concentration-dependent manner while promoting the expression of inducible hemoxygenase and peroxisome proliferator-activated receptor gamma (PPAR- $\gamma)$. The anti-inflammatory effects of Hedyotis diffusa are most likely facilitated by its inhibitory effects on the c-Jun N-terminal kinase (JNK) signaling pathway [16].

Radix Scrophulariae (Xuan Shen) is used to clear heat and cool the blood, nourish yin, and relieve toxicity. It is often used for the treatment of fever, swelling, neuritis, laryngitis, constipation, and inflammatory diseases [41]. The constituents of Radix Scrophulariae were reported to show antioxidant, antibacterial, anti-inflammatory, neuroprotective, antidiabetic, cytotoxic, and antimicrobial activities [17, 42]. In addition, the anti-inflammatory effects of this drug were associated with the NF-кB signaling pathway [43]. In another study, Radix Scrophulariae combined with Viticis Fructus was shown to decrease cell adhesion, alter the cell morphology to a round shape, and induce cell death in cancer cells [18].

Radix Ophiopogonis is capable of tonifying yin, engendering fluid, moistening the lungs, and clearing the heart; accordingly, it is often used for the treatment of dry cough, dry mouth, throat impediment and pain, vexation and insomnia, and constipation [44]. It has been shown to exert cardiovascular protection and anti-inflammatory, antidiabetic, anticancer, antioxidative, and immunomodulatory 
activities in previous studies $[45,46]$. The anti-inflammatory activity could be attributed to a decrease in IL-6 production [21]. In addition, this herb was found to induce apoptosis of prostate cancer cells via a receptor interacting serine/threonine kinase 1 (RIPK1)-related pathway both in vitro and in vivo [20].

We found that Gan-Lun-Yin and Zhi-Bai-Di-Huang-Wan were most commonly prescribed together, with Gan-Lun-Yin expected to nourish yin and clear heat and Zhi-Bai-Di-Huang-Wan expected to nourish the kidney and liver yin and clear heat [47, 48]. Both these CHPs clear heat and nourish yin, thus reducing the side effects of RT. The second most common combination was Gan-Lun-Yin and Xin-Yi-Qing-Fei-Tang; the former clears heat while the latter treats upper airway symptoms. With regard to single CHPs, Scutellariae Barbatae and Hedyotis diffusa were most commonly prescribed in combination. Both herbs can clear heat and show anticancer activities. The second most common combination was Radix Scrophulariae and Radix Ophiopogonis, both of which strengthen yin and reduce the side effects of RT. In summary, all these CHPs are generally prescribed in combination to enhance their efficacy, minimize toxicity, and tailor the treatment according to individual needs [49].

The top three formula CHPs and single CHPs in the present study share a common function of clearing heat, and most of them can nourish yin. The main treatment for NPC is RT, the side effects of which include mucositis, xerostomia, dysphagia, and 
dermatitis. All these side effects are a part of the heat syndrome as per TCM theory. Therefore, herbs that can clear heat and nourish yin are generally used to minimize RT-associated side effects. Although our findings were in accordance with TCM theory, well-designed clinical trials are required to further clarify our findings. At present, there is one prospective pilot study demonstrating that Gan-Lu-Yin can be used to treat dry mouth during RT for head and neck cancer, consistent with our findings [50].

This study has several limitations. First, we did not evaluate the efficacy and adverse effects of the CHPs. Although NHIRD has extensive prescription data, we did not have access to other chart-level records such as laboratory data and physician notes. Therefore, the usefulness of the treatments could not be assessed [49, 51]. Second, TCM is not fully covered by the NHI program in Taiwan. Moreover, visits to clinics not contracted with the NHI program were not included in the present study. In Taiwan, approximately 5\% TCM clinics are not contracted with the NHI program; therefore, TCM use may have been underestimated [25]. Third, patients with NPC show several different symptoms, and those in different stages and receiving different treatments develop variable complications [52]. Typically, TCM physician prescribe one or two formulae combined with several single herbs in one prescription based on the patient's symptoms. In the present study, however, we were unable to confirm the 
stage of cancer and treatments during CHP use. Therefore, further studies should evaluate the pattern of utilization of CHPs during different stages and treatments for cancer.

\section{Conclusions}

In conclusion, our findings suggest that CHPs are widely used for the management of NPC and its treatment-related side effects in Taiwan. TCM users with NPC are more likely to be women, younger individuals, white-collar workers, and residents of Central Taiwan. The most frequently prescribed CHPs for NPC are Gan-Lu-Yin and Hedyotis diffusa. These findings provide information regarding personalized therapies and can promote further clinical experiments and pharmacological research on CHPs for NPC treatment in Taiwan. Further well-designed, randomized, double-blind, placebo-controlled studies and basic mechanistic studies and clinical trials are needed to assess the safety and effectiveness of the prominent CHPs used for NPC management in Taiwan.

\section{Acknowledgement}

This work was supported by grants from the Ministry of Health and Welfare, Taiwan (MOHW107-TDU-B-212-123004), China Medical University Hospital, Academia 
Sinica Stroke Biosignature Project (BM10701010021), MOST Clinical Trial

Consortium for Stroke (MOST 106-2321-B-039-005), Tseng-Lien Lin Foundation,

Taichung, Taiwan, and Katsuzo and Kiyo Aoshima Memorial Funds, Japan. The

funders had no role in study design, data collection and analysis, decision to publish,

or preparation of the manuscript.

\section{References}

1. L. Tan; Loh, T. Benign and Malignant Tumors of the Nasopharynx, 6th ed.; Saunders, Ed. Elsevier Inc: 2015, pp. Number of 1420-1431.

2. M. L. K. Chua; Wee, J. T. S.; Hui, E. P.; Chan, A. T. C. Nasopharyngeal carcinoma. The Lancet 2016, 387, 1012-1024, doi:https://doi.org/10.1016/S0140-6736(15)00055-0.

3. W. I. Wei; Sham, J. S. Nasopharyngeal carcinoma. Lancet (London, England) 2005, 365, 2041-2054, doi:10.1016/s0140-6736(05)66698-6.

4. Y. Cao. EBV based cancer prevention and therapy in nasopharyngeal carcinoma. NPJ Precis Oncol 2017, 1, 10, doi:10.1038/s41698-017-0018-x.

5. S. W. Tsao; Tsang, C. M.; Lo, K. W. Epstein-Barr virus infection and nasopharyngeal carcinoma. Philos Trans R Soc Lond B Biol Sci 2017, 372, doi:10.1098/rstb.2016.0270.

6. Y. H. Lin. Utilisation and expenditure of radiotherapy among nasopharyngeal cancer patients in Taiwan. Clin Otolaryngol 2015, 40, 739-740, doi:10.1111/coa.12511.

7. C. N. Chu; Chen, P. C.; Bai, L. Y.; Muo, C. H.; Sung, F. C.; Chen, S. W. Young nasopharyngeal cancer patients with radiotherapy and chemotherapy are most prone to ischaemic risk of stroke: a national database, controlled cohort study. Clin Otolaryngol 2013, 38, 39-47, doi:10.1111/coa.12064.

8. G. K. Dy; Bekele, L.; Hanson, L. J.; Furth, A.; Mandrekar, S.; Sloan, J.; Adjei, A. A. Complementary and alternative medicine use by patients enrolled onto phase $I$ clinical trials. J Clin Oncol 2004, 22, 4810-4815, doi:10.1200/JCO.2004.03.121.

9. T. Zeller; Muenstedt, K.; Stoll, C.; Schweder, J.; Senf, B.; Ruckhaeberle, E.; Becker, S.; Serve, H.; Huebner, J. Potential interactions of complementary and alternative 
medicine with cancer therapy in outpatients with gynecological cancer in a comprehensive cancer center. J Cancer Res Clin Oncol 2013, 139, 357-365, doi:10.1007/s00432-012-1336-6.

10. S. M. Alsanad; Williamson, E. M.; Howard, R. L. Cancer patients at risk of herb/food supplement-drug interactions: a systematic review. Phytother Res 2014, 28, 1749-1755, doi:10.1002/ptr.5213.

11. Y. C. Chien; Sheu, M. J.; Wu, C. H.; Lin, W. H.; Chen, Y. Y.; Cheng, P. L.; Cheng, H. C. A Chinese herbal formula "Gan-Lu-Yin" suppresses vascular smooth muscle cell migration by inhibiting matrix metalloproteinase-2/9 through the PI3K/AKT and ERK signaling pathways. BMC Complement Altern Med 2012, 12, 137, doi:10.1186/1472-6882-12-137.

12. J. S. Yang; Wu, C. C.; Lee, H. Z.; Hsieh, W. T.; Tang, F. Y.; Bau, D. T.; Lai, K. C.; Lien, J. C.; Chung, J. G. Suppression of the TNF-alpha level is mediated by Gan-Lu-Yin (traditional Chinese medicine) in human oral cancer cells through the NF-kappa B, AKT, and ERK-dependent pathways. Environ Toxicol 2016, 31, 1196-1205, doi:10.1002/tox.22127.

13. T. Y. Laiabc; Kuo, H. W. Changes in urinary $\mathrm{Cu}, \mathrm{Zn}$, and Se levels in cancer patients after treatment with Sha Shen Mai Men Dong Tang. J Tradit Complement Med 2016, 6, 135-139, doi:10.1016/j.jtcme.2014.12.004.

14. C. Wu; Luo, H.; Ma, W.; Ren, X.; Lu, C.; Li, N.; Wang, Z. Polysaccharides isolated from Hedyotis diffusa inhibits the aggressive phenotypes of laryngeal squamous carcinoma cells via inhibition of Bcl-2, MMP-2, and muPA. Gene 2017, 637, 124-129, doi:10.1016/j.gene.2017.09.041.

15. Y. Jing; Kang, M.; Liu, J.; Li, J.; Tang, A. [Mechanism of apoptosis of nasopharyngeal carcinoma cells induced by polysaccharides extracts from Hedyotic diffusa]. Lin Chung Er Bi Yan Hou Tou Jing Wai Ke Za Zhi 2015, 29, 641-644.

16. Y. Xu; Chen, X. X.; Jiang, Y. X.; Zhang, D. D. Ethyl Acetate Fraction from Hedyotis diffusa plus Scutellaria barbata Exerts Anti-Inflammatory Effects by Regulating miR-155 Expression and JNK Signaling Pathway. Evid Based Complement Alternat Med 2018, 2018, 3593408, doi:10.1155/2018/3593408.

17. J. Hua; Qi, J.; Yu, B. Y. Iridoid and phenylpropanoid glycosides from Scrophularia ningpoensis Hemsl. and their alpha-glucosidase inhibitory activities. Fitoterapia 2014, 93, 67-73, doi:10.1016/j.fitote.2013.11.011.

18. A. Kim; Im, M.; Ma, J. Y. SRVF, a novel herbal formula including Scrophulariae Radix and Viticis Fructus, disrupts focal adhesion and causes detachment-induced apoptosis in malignant cancer cells. Sci Rep 2017, 7, 12756, doi:10.1038/s41598-017-12934-y. 
19. C. C. Zhang; Gu, W. L.; Wu, X. M.; Li, Y. M.; Chen, C. X.; Huang, X. Y. Active components from Radix Scrophulariae inhibits the ventricular remodeling induced by hypertension in rats. Springerplus 2016, 5, 358, doi:10.1186/s40064-016-1985-z.

20. Z. Lu; Wang, H.; Zhu, M.; Song, W.; Wang, J.; Wu, C.; Kong, Y.; Guo, J.; Li, N.; Liu, J., et al. Ophiopogonin D', a Natural Product From Radix Ophiopogonis, Induces in Vitro and in Vivo RIPK1-Dependent and Caspase-Independent Apoptotic Death in Androgen-Independent Human Prostate Cancer Cells. Front Pharmacol 2018, 9, 432, doi:10.3389/fphar.2018.00432.

21. Y. Kitahiro; Koike, A.; Sonoki, A.; Muto, M.; Ozaki, K.; Shibano, M. Anti-inflammatory activities of Ophiopogonis Radix on hydrogen peroxide-induced cellular senescence of normal human dermal fibroblasts. J Nat Med 2018, 10.1007/s11418-018-1223-9, doi:10.1007/s11418-018-1223-9.

22. Y. T. Kuo; Chang, T. T.; Muo, C. H.; Wu, M. Y.; Sun, M. F.; Yeh, C. C.; Yen, H. R. Use of Complementary Traditional Chinese Medicines by Adult Cancer Patients in Taiwan: A Nationwide Population-Based Study. Integr Cancer Ther 2018, 17, 531-541, doi:10.1177/1534735417716302.

23. L. Nancy; Riaz, N.; Ove, R.; Reyngold, M. L.; Foote, R. L.; Bonner, J. A. Nasopharyngeal Carcinoma, fourth ed.; , 2016, pp. Number of 629-648.

24. W. C. Cho; Chen, H. Y. Clinical efficacy of traditional Chinese medicine as a concomitant therapy for nasopharyngeal carcinoma: a systematic review and meta-analysis. Cancer Invest 2009, 27, 334-344, doi:10.1080/07357900802392683.

25. C. M. Chang; Chu, H. T.; Wei, Y. H.; Chen, F. P.; Wang, S.; Wu, P. C.; Yen, H. R.; Chen, T. J.; Chang, H. H. The Core Pattern Analysis on Chinese Herbal Medicine for Sjogren's syndrome: A Nationwide Population-Based Study. Sci Rep 2015, 5, 9541, doi:10.1038/srep09541.

26. C. Kai; Puzhi, L.; Ruyi, C. Clinical analysis of Ganluyin drink on injure of radioactive oral mucosa causedby super branch radiotherapies, which treats cancer of nasopherynx. Hebei J TCM 2000, 22.

27. T. Kumagai; M“uller, C. I.; Desmond, J. C.; Imai, Y.; Heberb, D.; Koeffler, H. P. Scutellaria baicalensis, a herbal medicine: anti-proliferative and apoptotic activity against acute lymphocytic leukemia, lymphoma and myeloma cell lines. Leuk Res 2007, 31, 523-530, doi:10.1016/j.leukres.2006.08.019.

28. F. Ye; Jiang, S.; Volshonok, H.; Wu, J.; Zhang, D. Y. Molecular mechanism of anti-prostate cancer activity of Scutellaria baicalensis extract. Nutr Cancer 2007, 57, 100-110, doi:10.1080/01635580701268352.

29. J. C.-J. Chao; Chiang, S. W.; Wang, C. C.; Tsai, Y.-H.; Wu, M.-S. Hot water-extracted 
Lycium barbarum and Rehmannia glutinosa inhibit proliferation and induce apoptosis of hepatocellular carcinoma cells. World J Gastroenterol 2006, 12, 4478-4484.

30. C. H. Pan; Hsieh, I. C.; Liu, F. C.; Hsieh, W. T.; Sheu, M. J.; Koizumi, A.; Wu, C. H. Effects of a Chinese herbal health formula, "Gan-Lu-Yin", on angiogenesis. J Agric Food Chem 2010, 58, 7685-7692, doi:10.1021/jf1002254.

31. H. R. Yen; Sun, M. F.; Lin, C. L.; Sung, F. C.; Wang, C. C.; Liang, K. L. Adjunctive traditional Chinese medicine therapy for patients with chronic rhinosinusitis: a population-based study. Int Forum Allergy Rhinol 2015, 5, 240-246, doi:10.1002/alr.21446.

32. H. R. Yen; Liang, K. L.; Huang, T. P.; Fan, J. Y.; Chang, T. T.; Sun, M. F. Characteristics of traditional Chinese medicine use for children with allergic rhinitis: a nationwide population-based study. Int J Pediatr Otorhinolaryngol 2015, 79, 591-597, doi:10.1016/j.ijporl.2015.02.002.

33. J. Dou; Wang, Z.; Ma, L.; Peng, B.; Mao, K.; Li, C.; Su, M.; Zhou, C.; Peng, G. Baicalein and baicalin inhibit colon cancer using two distinct fashions of apoptosis and senescence. Oncotarget 2018, 9, 20089-20102, doi:10.18632/oncotarget.24015.

34. D. H. KIM; HOSSAIN, M. A.; KANG, Y. J.; JANG, J. Y.; LEE, Y. J.; IM, E.; YOON, J.-H.; KIM, H. S.; CHUNG, H. Y.; KIM1, N. D. Baicalein, an active component of Scutellaria baicalensis Georgi, induces apoptosis in human colon cancer cells and prevents AOM/DSS-induced colon cancer in mice. Int J Oncol 2013, 43, 1652-1658, doi:10.3892/ijo.2013.2086.

35. X. Yu; Yang, Y.; Li, Y.; Cao, Y.; Tang, L.; Chen, F.; Xia, J. Baicalein inhibits cervical cancer progression via downregulating long noncoding RNA BDLNR and its downstream PI3K/Akt pathway. Int J Biochem Cell Biol 2018, 94, 107-118, doi:10.1016/j.biocel.2017.11.009.

36. C. Lu; Wang, H.; Chen, S.; Yang, R.; Li, H.; Zhang, G. Baicalein inhibits cell growth and increases cisplatin sensitivity of A549 and H460 cells via miR-424-3p and targeting PTEN/PI3K/Akt pathway. J Cell Mol Med 2018, 22, 2478-2487, doi:10.1111/jcmm.13556.

37. D. Sung, Dong, C.D. Treatment of oral dryness using SMD-2 among head and neck cancer patients. haanxi J Chin Med 2001, 27, 54.

38. A. K. Maitra; Dorani, B. Role of zinc in post-injury wound healing. Arch Emerg Med 1992, 9, 122-124.

39. Y. Y. Cheng; Hsieh, C. H.; Tsai, T. H. Concurrent administration of anticancer chemotherapy drug and herbal medicine on the perspective of pharmacokinetics. J Food Drug Anal 2018, 26, S88-S95, doi:10.1016/j.jfda.2018.01.003. 
40. J. Lin; Li, Q.; Chen, H.; Lin, H.; Lai, Z.; Peng, J. Hedyotis diffusa Willd. extract suppresses proliferation and induces apoptosis via IL-6-inducible STAT3 pathway inactivation in human colorectal cancer cells. Oncol Lett 2015, 9, 1962-1970, doi:10.3892/ol.2015.2956.

41. L. J. Zhu; Hou, Y. L.; Shen, X. Y.; Pan, X. D.; Zhang, X.; Yao, X. S. Monoterpene pyridine alkaloids and phenolics from Scrophularia ningpoensis and their cardioprotective effect. Fitoterapia 2013, 88, 44-49, doi:10.1016/j.fitote.2013.04.005.

42. Q. J. Ma; Han, L.; Mu, Y.; Guan, P. P.; Lei, H.; Wang, Z. Y.; Huang, X. S. New Iridoids from Scrophularia ningpoensis. Chem Pharm Bull (Tokyo) 2017, 65, 869-873, doi:10.1248/cpb.c17-00163.

43. S. Y. Sheu; Hong, Y. W.; Sun, J. S.; Liu, M. H.; Chen, C. Y.; Ke, C. J. Radix Scrophulariae extracts (harpagoside) suppresses hypoxia-induced microglial activation and neurotoxicity. BMC Complement Altern Med 2015, 15, 324, doi:10.1186/s12906-015-0842-x.

44. J. Fang; Wang, X.; Lu, M.; He, X.; Yang, X. Recent advances in polysaccharides from Ophiopogon japonicus and Liriope spicata var. prolifera. Int J Biol Macromol 2018, 114, 1257-1266, doi:10.1016/j.ijbiomac.2018.04.022.

45. M. H. Chen; Chen, X. J.; Wang, M.; Lin, L. G.; Wang, Y. T. Ophiopogon japonicus--A phytochemical, ethnomedicinal and pharmacological review. J Ethnopharmacol 2016, 181, 193-213, doi:10.1016/j.jep.2016.01.037.

46. N. Li; Zhang, J. Y.; Zeng, K. W.; Zhang, L.; Che, Y. Y.; Tu, P. F. Anti-inflammatory homoisoflavonoids from the tuberous roots of Ophiopogon japonicus. Fitoterapia 2012, 83, 1042-1045, doi:10.1016/j.fitote.2012.05.011.

47. H. C. Lai; Lin, H. J.; Huang, S. T. Chinese herbal medicine to treat urolithiasis in a patient with right flank pain and hematuria: A case report. Complement Ther Med 2018, 36, 118-122, doi:10.1016/j.ctim.2017.12.007.

48. S. K. Lin; Lin, P. H.; Hsu, R. J.; Chuang, H. C.; Liu, J. M. Traditional Chinese medicine therapy reduces the catheter indwelling risk in dementia patients with difficult voiding symptoms. J Ethnopharmacol 2017, 203, 120-126, doi:10.1016/j.jep.2017.03.040.

49. Y. C. Hung; Kao, C. W.; Lin, C. C.; Liao, Y. N.; Wu, B. Y.; Hung, I. L.; Hu, W. L. Chinese Herbal Products for Female Infertility in Taiwan: A Population-Based Cohort Study. Medicine (Baltimore) 2016, 95, e3075, doi:10.1097/MD.0000000000003075.

50. P. Y. Hsu; Yang, S. H.; Tsang, N. M.; Fan, K. H.; Hsieh, C. H.; Lin, J. R.; Hong, J. H.; Lin, Y. C.; Chen, H. Y.; Yang, C. T., et al. Efficacy of Traditional Chinese Medicine in Xerostomia and Quality of Life during Radiotherapy for Head and Neck Cancer: A 
Prospective Pilot Study. Evid Based Complement Alternat Med 2016, 2016, 8359251, doi:10.1155/2016/8359251.

51. Y. N. Liao; Hu, W. L.; Chen, H. J.; Hung, Y. C. The Use of Chinese Herbal Medicine in the Treatment of Chronic Obstructive Pulmonary Disease (COPD). Am J Chin Med 2017, 45, 225-238, doi:10.1142/S0192415X17500148.

52. F. E. Mott; Ferrarotto, R.; Nguyen, T.; Phan, J. Nasopharyngeal carcinoma outcome with induction chemotherapy followed by concurrent chemoradiotherapy. Oral Oncol 2018, 81, 75-80, doi:10.1016/j.oraloncology.2018.04.020. 